\title{
Fatores de risco associados ao comportamento sexual de adolescentes
}

\author{
Risk factors associated with sexual adolescent behavior
}

Factores de riesgo asociados con el comportamiento adolescente sexual

Jackeline Kérollen Duarte de Sales ${ }^{1}$, Janayle Kéllen Duarte de Sales ${ }^{2 *}$, Dailon de Araujo Alves ${ }^{1}$, Hercules Pereira Coelho², Ozeias Pereira de Oliveira ${ }^{3}$, Rosely Leyliane dos Santos ${ }^{1}$.

\section{RESUMO}

Objetivo: Identificar fatores de risco associados ao comportamento sexual de adolescentes. Métodos: Tratase de um estudo do tipo revisão integrativa da literatura, desenvolvido em seis etapas, realizado através da busca indexada de artigos nas bases de dados da LILACS, BDENF, PUBMED e SCIELO, com os seguintes descritores: "Adolescente" AND "Comportamento sexual" AND "Fatores de risco". Foram utilizados estudos disponíveis na íntegra, do tipo artigo científico, publicados entre os anos de 2013 a 2018, nos idiomas inglês, português e espanhol. Resultados: Os principais fatores de risco encontrados para o comportamento sexual dos adolescentes foram: o uso de álcool, tabaco e outras drogas, o não uso de preservativo, ter múltiplos parceiros, sexarca precoce e o não conhecimento e informações sobre o tema. Para discutir os fatores de risco evidenciados, elegeram-se três categorias: Comportamento Sexual: conhecimentos, condutas e outros fatores associados; Álcool, Tabaco e outras Drogas: repercussões de risco para o comportamento sexual; e Infecções Sexualmente Transmissíveis em Adolescentes. Considerações Finais: Diante dos achados, ações estratégicas, como por exemplo a educação em saúde, podem ser desenvolvidas como intervenção neste grupo, e conduzidas de forma mais pontual, para obtenção de resultados significativos.

Palavras-chave: Adolescente, Comportamento sexual, Fatores de risco.

\begin{abstract}
Objective: To identify risk factors associated with the sexual behavior of adolescents. Methods: This is an integrative literature review study, developed in six stages, carried out through the indexed search of articles in the databases of LILACS, BDENF, PUBMED and SCIELO, with the following descriptors: "Adolescent" AND "Sexual behavior" AND "Risk factors". Have been used studies fully available scientific article type, were used between the years 2013 to 2018, in English, Portuguese and Spanish. Results: The main risk factors found for the sexual behavior of adolescents were: the use of alcohol, tobacco and other drugs, the non-use of condoms, having multiple partners, early sexarche and the lack of knowledge and information on the subject. To discuss the evidenced risk factors, three categories were chosen: Sexual Behavior: knowledge, behavior and other associated factors; Alcohol, Tobacco and other Drugs: risk repercussions for sexual behavior; and Sexually Transmitted Infections in Adolescents. Final Considerations: In view of the findings, strategic actions, such as health education, can be developed as an intervention in this group, and conducted more punctually, to obtain significant results.
\end{abstract}

Key words: Adolescent, Sexual behavior, Risk factors.

\footnotetext{
${ }^{1}$ Universidade Regional do Cariri (URCA). Crato - Ceará.

*E-mail: janayleduarte@gmail.com

${ }^{2}$ Centro Universitário Doutor Leão Sampaio (UNILEÃO). Juazeiro do Norte - Ceará.

${ }^{3}$ Faculdades Integradas de Patos (FIP). Crato - Ceará.
} 


\section{RESUMEN}

Objetivo: identificar los factores de riesgo asociados con el comportamiento sexual de los adolescentes. Métodos: Este es un estudio integrador de revisión de literatura, desarrollado en seis etapas, llevado a cabo mediante la búsqueda indexada de artículos en las bases de datos de LILACS, BDENF, PUBMED y SCIELO, con los siguientes descriptores: "Adolescente" Y " Comportamiento sexual "Y" Factores de riesgo ". Los estudios disponibles en su totalidad, del tipo de artículo científico, se utilizaron entre los años 2013 a 2018, en inglés, portugués y español. Resultados: Los principales factores de riesgo encontrados para el comportamiento sexual de los adolescentes fueron: el uso de alcohol, tabaco y otras drogas, el no uso de condón, tener múltiples parejas, el sexarca temprano y la falta de conocimiento e información sobre el tema. Para discutir los factores de riesgo evidenciados, se eligieron tres categorías: Comportamiento Sexual: conocimiento, conductas y otros factores asociados; Alcohol, Tabaco y otras Drogas: repercusiones de riesgo para el comportamiento sexual; e Infecciones de Transmisión Sexual en Adolescentes. Consideraciones finales: en vista de los hallazgos, las acciones estratégicas, como la educación para la salud, pueden desarrollarse como una intervención en este grupo, y llevarse a cabo de manera más puntual para obtener resultados significativos.

Palabras clave: Adolescente, Comportamiento sexual, Factores de riesgo.

\section{INTRODUÇÃO}

A fase da adolescência é definida pela Organização Mundial de Saúde (OMS) como o período transcorrido dos 10 aos 19 anos de idade, no qual, geralmente, se inicia a atividade sexual (GONÇALVES H, et al., 2015). A adolescência é uma fase da vida permeada por diversas mudanças psicofisiológicas, em que o corpo sofre atuação de hormônios que intensificam a sexualidade.

Associado a isto, iniciam-se novas descobertas psicossociais que podem influenciar as experimentações de comportamentos que, por vezes, são desmedidos de segurança (CHINAZZO IR, et al., 2014).

A adolescência tornou-se evidente tema para estudos a partir dos problemas sociais que caracterizam esta fase da vida, dentre os quais podemos citar os comportamentos de riscos, que por vezes, acabam por comprometer a saúde do adolescente, evidenciando-se assim, como um problema de saúde pública que carece de pesquisas e políticas públicas eficazes (BRASIL, 2017).

Os comportamentos de risco são condutas que os indivíduos desenvolvem, e que, por conseguinte, podem comprometer a sua saúde. Estas atitudes podem estar diretamente relacionadas às esferas de âmbito psicológico, intelectual, social, cultural e com o nível socioeconômico (SASAKI RSA, et al., 2014; GONÇALVES H, et al., 2015).

Neste contexto, a exposição a estes fatores de risco pode cominar na realização de condutas de risco, e na exposição a diferentes consequências, desde sociais a problemas de saúde (REIS AAC, et al., 2018). Alguns desses fatores de risco estão associados ao desenvolvimento de Doenças Crônicas Não Transmissíveis (DCNT), acidentes, violências e gravidez indesejada.

A Pesquisa Nacional de Saúde do Escolar (PeNSE), realizada em 2012 com escolares no Brasil, demonstrou que $25 \%$ dos adolescentes que já tiveram relações sexuais não usaram preservativos (CAMPOS MO, et al., 2014). Como consequência, dados estatísticos de 1980 a 2009 revelam que, entre os adolescentes e jovens, o Brasil tem proporcionalmente o triplo do percentual de casos da Síndrome da Imunodeficiência Adquirida (AIDS), se comparado à França (TAQUETTE SR, 2013).

Ao passo que a taxa de incidência de gravidez na adolescência no Brasil revela-se acima da média, com $68,4 / 1.000$ meninas, enquanto a taxa mundial é de 46,0/1.000 (OPAS, 2018). A complexidade desses comportamentos de risco podem gerar no adolescente diversos problemas relacionados à violência consigo mesmo, no que tange sua exposição a riscos que podem comprometer a sua própria saúde, o que caracteriza tal aspecto como uma preocupação para a saúde pública, uma vez que estes comportamentos são 
associados a diversos fatores, muitos ainda desconhecidos, em razão da carência de estudos acerca da temática, e da inexistência de incentivos públicos para promoção da orientação sexual nas escolas (AGOSTINI A, et al., 2014).

Tendo em vista essa problemática, o estudo justifica-se pela necessidade de reconhecer e identificar os fatores de risco que podem comprometer o comportamento sexual de adolescentes, o que possibilita a criação de estratégias efetivas para a atuação de profissionais da saúde, e o estabelecimento de políticas públicas eficazes. Assim, tem-se como objetivo do estudo identificar os fatores de risco associados ao comportamento sexual de adolescentes.

\section{MÉTODOS}

Trata-se de uma revisão integrativa da literatura, que é um método científico que busca sintetizar o conhecimento sobre um assunto específico, identificar lacunas em pesquisas, e sugerir novos estudos (MENDES KDS, et al., 2019).

O estudo foi desenvolvido em observância às etapas propostas por Souza MT, et al. (2010), os quais fragmentam o desenvolvimento de pesquisas do tipo revisão integrativa em seis etapas, conforme elucidado a seguir.

A primeira fase do estudo foi a elaboração da pergunta norteadora, concretizada por meio de leituras prévias e questionamentos, com o objetivo de aprimorar a prática clínica em saúde do adolescente. Para isso, buscou-se responder a seguinte indagação: Quais fatores de risco estão associados ao comportamento sexual de adolescentes?

O segundo momento do estudo foi à busca da amostragem na literatura, realizada nas bases de dados da Literatura Latino-Americana e do Caribe em Ciências da Saúde (LILACS), Base de Dados em Enfermagem (BDENF), U.S. National Library of Medicine (PUBMED), e na Scientific Electronic Library Online (SCIELO), por meio do cruzamento dos Descritores em Ciências da Saúde "Adolescente" AND "Comportamento sexual" AND "Fatores de risco".

Definiram-se como critérios de inclusão: estudos disponíveis na íntegra, do tipo artigo científico, publicados entre os anos de 2013 a 2018, nos idiomas inglês, português e espanhol. Ao passo que foram excluídos da amostragem os estudos que não se adequavam ao tema proposto e/ou não respondiam à questão do estudo, revisões bibliográficas da literatura, e pesquisas duplicadas nas bases de dados.

A terceira etapa da revisão deu-se a partir da coleta de dados, a partir da qual foi elaborado um banco de dados, e, por conseguinte, realizada a codificação e a categorização dos estudos.

Para organização e sintetização das informações foi utilizado uma tabela contendo a codificação dos estudos, ano e autoria, título do estudo, objetivo principal e resultados. Ressalta-se que foram realizados fichamentos de todos os artigos incluídos na amostra, a fim de promover uma maior acurácia na extração das informações significativas.

A quarta fase foi a análise crítica dos estudos incluídos, na qual os artigos foram avaliados criticamente, buscando-se estabelecer aspectos em comum, e averiguar as divergências, a partir dos quais foram elaborados os resultados deste estudo.

No quinto passo, discussão dos resultados, foi desenvolvida a interpretação e discussão dos resultados com a literatura pertinente ao assunto. A partir da qual, destacaram-se as recomendações e os fatores de risco relatados nos estudos e sugestões de pesquisas futuras.

A última etapa da revisão constituiu-se a partir da construção desse estudo, apresentação da revisão e síntese do conhecimento.

O fluxograma da coleta de dados é apresentado na (Figura 1), o qual demonstra as etapas percorridas e o passo a passo para a seleção e organização dos artigos que compuseram o estudo. 
Figura 1 - Fluxograma da seleção dos estudos de acordo com o Preferred Reporting Items for Systematic Reviews and Meta-Analyses (PRISMA). Crato - Ceará. Brasil. 2019.
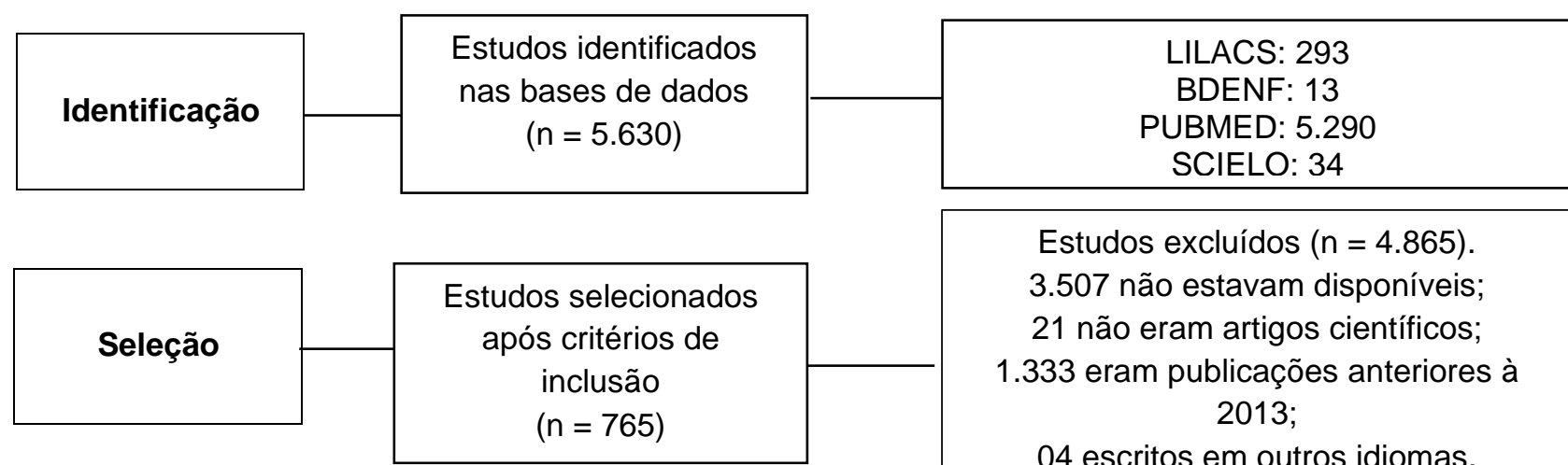

Estudos excluídos $(\mathrm{n}=4.865)$.

3.507 não estavam disponíveis;

21 não eram artigos científicos;

1.333 eram publicações anteriores à 2013;

04 escritos em outros idiomas.

Estudos excluídos $(\mathrm{n}=703)$.

634 não se adequavam ao tema;

55 duplicados;

14 artigos de revisão.

$$
(n=62)
$$

40 excluídos por abordarem outra população, e/ou que não respondiam questão da pesquisa.

Fonte: Sales JKD, et al., 2019.

\section{RESULTADOS E DISCUSSÃO}

Diante dos estudos selecionados, mediante a leitura dos artigos na íntegra, foram estabelecidas as categorias temáticas, e listados os principais fatores de risco associados ao comportamento sexual dos adolescentes, de acordo com as menções dos estudos analisados pelos autores (Tabela 1). 
Tabela 1 - Fatores de risco associados ao comportamento sexual de adolescentes. Crato, Ceará. Brasil. 2019.

\begin{tabular}{|c|c|c|}
\hline Fator de risco evidenciado & Descrição & Citações \\
\hline $\begin{array}{l}\text { Álcool, Tabaco e } \\
\text { outras drogas }\end{array}$ & $\begin{array}{l}\text { Incluem álcool, tabaco e drogas ilícitas, } \\
\text { como maconha e cocaína. }\end{array}$ & 12 estudos \\
\hline Não uso de preservativo & $\begin{array}{l}\text { Refere-se à relação sexual sem proteção, } \\
\text { do tipo camisinha. }\end{array}$ & 12 estudos \\
\hline Múltiplos parceiros & $\begin{array}{l}\text { Adolescentes que tiveram, e têm relações } \\
\text { sexuais com mais de um parceiro. }\end{array}$ & 7 estudos \\
\hline $\begin{array}{l}\text { Falta de conhecimento e } \\
\text { informação }\end{array}$ & $\begin{array}{l}\text { Relaciona-se ao saber dos adolescentes } \\
\text { sobre proteção, contracepção, e Infecções } \\
\text { Sexualmente Transmissíveis (IST). }\end{array}$ & 7 estudos \\
\hline Sexarca & $\begin{array}{l}\text { Envolve todos os aspectos relacionados à } \\
\text { primeira relação sexual, incluindo a idade } \\
\text { em que ocorreu. }\end{array}$ & 6 estudos \\
\hline $\begin{array}{l}\text { Baixa condição } \\
\text { socioeconômica }\end{array}$ & $\begin{array}{l}\text { Baixa condição econômica e social da } \\
\text { família. }\end{array}$ & 5 estudos \\
\hline Relacionamento afetivo & $\begin{array}{l}\text { Relacionamento com parceiro, que pode } \\
\text { gerar confiança e envolvimento emocional. }\end{array}$ & 4 estudos \\
\hline Violência física/emocional & $\begin{array}{c}\text { Descrito como qualquer tipo de violência } \\
\text { psicológica, ou de agressão física, inclusive } \\
\text { estrupo. }\end{array}$ & 3 estudos \\
\hline Saúde mental prejudicada & $\begin{array}{l}\text { Foram considerados o estado depressivo, } \\
\text { tristeza e ideação suicida. }\end{array}$ & 3 estudos \\
\hline Inatividade física & Prática irregular de atividade física & 2 estudos \\
\hline Relação homossexual & $\begin{array}{l}\text { Relação que envolve parceiro do mesmo } \\
\text { sexo. }\end{array}$ & 2 estudos \\
\hline Idade do parceiro & A idade do parceiro sexual. & 2 estudos \\
\hline $\begin{array}{l}\text { Experiência traumática; } \\
\text { relacionamento familiar } \\
\text { instável; baixa escolaridade; } \\
\text { residir em zona rural/urbana; e } \\
\text { crenças religiosas. }\end{array}$ & $\begin{array}{l}\text { Fatores que foram tratados em apenas um } \\
\text { estudo, sem desenvolvimento do tema. }\end{array}$ & $\begin{array}{l}\text { Pelo menos um } \\
\text { estudo. }\end{array}$ \\
\hline
\end{tabular}

Fonte: Sales JKD, et al., 2019.

Os estudos revelaram que os principais fatores de risco para o comportamento sexual foi o uso de álcool, tabaco e outras drogas, o não uso de preservativo, ter múltiplos parceiros, sexarca precoce e a carência de conhecimento e informações sobre o tema. Para discutir os fatores de risco evidenciados, elegeram-se três categorias: Comportamento Sexual: conhecimentos, condutas e outros fatores associados; Álcool, Tabaco e outras Drogas: repercussões de risco para o comportamento sexual; e Infecções Sexualmente Transmissíveis em Adolescentes.

\section{Comportamento sexual: conhecimentos, condutas e outros fatores associados}

Quando se trata de comportamento sexual de risco a literatura aponta diferentes definições, o que dificulta a realização de comparações entre resultados. A partir da análise dos dados averiguou-se que alguns fatores tratados, comumente, como características do comportamento sexual, são abordados em outros estudos como componentes do comportamento sexual de risco (GRAF DD, et al., 2020). Assim, compreender o comportamento sexual dos adolescentes permite identificar as características que indicam risco e os fatores que podem estar associados. O conhecimento e as condutas inadequadas podem expor os adolescentes ao risco de IST. Neste contexto, é importante ressaltar que apesar dos pais serem importantes fontes de 
informação sobre assuntos sexuais, os mesmos não são reconhecidos como fonte de informação, em detrimento da carência de comunicação sobre sexualidade na adolescência (VISALLI G, 2019). Fato este que incita forte potencial para aumentar o risco, essencialmente quando a referência não é clara, ou é incorreta.

Os adolescentes buscam recurso à contracepção na primeira relação sexual, contudo a quantidade de adolescentes que não utilizam nenhum método contraceptivo nas relações subsequentes, por considerarem desnecessário, é preocupante. Estudos contemporâneos afirmam ainda que, comumente, os adolescentes conduzem suas relações sexuais, por vezes, sob o efeito de substâncias psicoativas (MIRANDA PSF, et al., 2018).

Resultados similares foram apontados no estudo de Graf DD, et al. (2020), os quais evidenciaram que os estudantes realizaram uso de substâncias psicoativas antes da última relação sexual.As adolescentes sexualmente ativas têm mais probabilidades de relatar o não uso de contracepção do que os meninos. O sexo feminino apresentou maior probabilidade de ter um comportamento sexual de risco, principalmente quando associado a fatores de autoestima e relação familiar (CASOLA AR, et al., 2017; FARID ND, et al., 2014; SANCHEZ ZM, et al., 2013). Resultado este que se contrapõe ao dos meninos, os quais, frequentemente, são influenciados pelo fator de risco do álcool, tabaco e outras drogas.

Contudo, os adolescentes do sexo masculino apresentam mais condutas de risco do que as adolescentes do sexo feminino, dados estes averiguados a partir da maior exposição aos fatores de risco, dentre eles: o uso de álcool, tabaco e outras drogas, violência física/emocional, sexarca precoce e multiplicidade de parceiros (COELHO MEGM, et al., 2017).

A intenção de realizar de relações sexuais sem preservativo associa-se aos aspectos emocionais e cognitivos. $\mathrm{O}$ aspecto sociocognitivo foi o preditor mais prevalente diante da intenção de voltar a realizar o comportamento de risco, relação sexual sem preservativo (CHINAZZO IR, et al., 2014). O comportamento homossexual e bissexual associou-se de forma mais prevalente aos fatores de risco à saúde: ter se "embriagado" no último ano; ter usado drogas ilícitas no último ano; não ter usado camisinha durante as relações sexuais; ter tido experiências sexuais traumáticas quando era criança ou adolescente; e ter ideação suicida, do que o comportamento heterossexual, entre os adolescentes escolares (CHINAZZO IR, et al., 2014).

Adolescentes em situação de rua estão mais predispostos aos comportamentos de risco, devido à baixa percepção de risco, múltiplos parceiros, compartilhamento de objetos pessoais, desnutrição e assédio sexual (GONÇALVES H, et al., 2015). Há uma estreita relação entre a iniciação sexual em menores de 14 anos, com comportamentos vulneráveis à saúde, experimentação de álcool e fumo, episódio de embriaguez, envolvimento em brigas e uso de drogas ilícitas. Os meninos revelaram maior número de parceiros sexuais, que as meninas (SASAKI RSA, et al., 2014; MESQUITA JS, et al., 2017). Neste contexto, os adolescentes que tiveram uma sexarca precoce relataram um maior uso de substâncias na adolescência do que os jovens que iniciaram sua vida sexual de maneira tardia e/ou que ainda não tiveram relações sexuais.

Apontado como um modesto preditor, a estreia sexual, ainda na adolescência, está associada a uma maior experimentação de substâncias, frequência e intenção de uso, e acesso a substâncias (CLARK DA, et al., 2020). Ressalta-se que esse grupo etário referiu também a maior probabilidade de ter outros comportamentos de risco, como fumar, usar álcool e drogas ilícitas (maconha e anfetaminas) (SHAYO FK e KALOMO MH, 2019). Ao passo que Gutierrez EB, et al. (2019) afirmam que mulheres que tiveram o início sexual tardio são associadas ao sexo protegido durante o último intercurso sexual. Os adolescentes revelaram em suas falas fatores de risco como o desconhecimento sobre as IST, sexo sem proteção, envolvimento afetivo com o parceiro, o uso de drogas ilícitas, relação familiar desestruturada, e baixas condições socioeconômicas e de escolaridade.

Sendo os fatores de proteção ter um parceiro confiável e um relacionamento com diálogo aberto, uso de preservativo, diálogo com professores e amigos, e a participação dos pais em ações na comunidade, junto com os adolescentes, com o objetivo de favorecer a compreensão dos mesmos quanto às medidas de prevenção das IST, e demais problemas advindos da exposição aos supracitados fatores de risco (PATIAS ND e DIAS ACG, 2014).

A conexão com os pais, pela adolescente do sexo feminino, revelou ser um fator significante que a torna menos propensa a ter relações sexuais e/ou múltiplos parceiros. Essa comunicação familiar, associada à 
conexão parental, fornece proteção contra comportamentos de risco entre os adolescentes (SHAYO FK e KALOMO MH, 2019). As pesquisas indicam que esse envolvimento dos pais e familiares afeta 0 comportamento dos adolescentes principalmente por meio do monitoramento (GEBREYESUS H, 2019).

O uso de contraceptivos nas relações subsequentes a sexarca, motivações pessoais e crenças errôneas sobre a contracepção estão associados à vulnerabilidade dos adolescentes à ocorrência de gravidez indesejada (MOURA LR, et al., 2013). Observou-se também que não houve nenhuma associação estatisticamente significativa entre o conhecimento dos adolescentes sobre as IST e o comportamento sexual de risco, não uso de preservativo e multiplicidade de parceiros (PIMENTEL JC, et al., 2017).

Além do uso de contraceptivos de maneira irregular, alguns fatores podem aumentar a probabilidade de gravidez na adolescência, dentre os quais podemos citar: multiplicidade de parceiros e atividade sexual frequente (OCHEN AM, et al., 2019). Comparando as práticas regulares de atividade física, como lazer e a prática nas aulas de educação física, revela-se que houve uma maior exposição aos fatores associados ao comportamento sexual de risco, não uso de preservativo e múltiplos parceiros, naqueles que praticam atividade física por meio das aulas da disciplina supracitada (MAIA DAC, et al., 2018).

Diante dessa perspectiva de comportamento sexual de risco, demonstra-se que são necessárias políticas de institucionalização da educação sexual nas escolas, assim como a atualização de conceitos e do repertório de fatores que podem impactar nos comportamentos sexuais dos adolescentes (GRAF DD, et al., 2020).

O acesso a preservativos de maneira fácil e gratuita, foi apontado como uma importante medida para 0 desenvolvimento de relações sexuais seguras (GUTIERREZ EB, et al., 2019). Ressalta-se que, os profissionais da saúde devem expandir a maneira como preenchem essas lacunas (educação sexual) durante o início da adolescência e o final da meia-idade (LINDBERG LD, et al., 2019).

\section{Álcool, Tabaco e outras Drogas: repercussões de risco para o comportamento sexual}

Os adolescentes têm curiosidade em explorar o desconhecido e aventurar-se por uma série de comportamentos de risco, incluindo relações sexuais e o uso de substâncias.

O paralelismo entre as relações sexuais e outros comportamentos de risco incita que há um agrupamento de fatores que predispõe riscos à saúde entre os adolescentes. Fato este, exposto pela correlação entre os comportamentos de risco e o uso de substâncias, o que demonstrou conduzir os comportamentos sexuais de risco entre adolescentes (SHAYO FK e KALOMO MH, 2019).

O uso de Álcool, tabaco e outras drogas, principalmente as ilícitas, foram os fatores de risco mais relatados pela literatura. $O$ uso de álcool por adolescentes, geralmente, ocorre em contextos que aumentam a exposição dos mesmos ao risco sexual. De acordo com Livingston JA, et al. (2015), quando a primeira relação sexual acontece associada ao uso do álcool, há uma tendência para que as relações subsequentes também sejam realizadas sob efeitos alcoólicos.

Alguns aspectos que envolvem a sexarca influem diretamente nas demais relações sexuais. Quando a estreia sexual acontece de forma precoce, há tendências gerais que predispõem ao desenvolvimento e exposição do adolescente a uma ampla gama de comportamentos problemáticos, tais como comportamento sexual de risco, uso de substâncias e outros (CLARK DA, et al., 2020).

Adolescentes sexualmente ativos que, frequentemente, já haviam experimentado álcool, relataram terem se arrependido das relações sexuais que tiveram sob efeito deste, e até relataram que não terem utilizado o preservativo (REIS TG e OLIVEIRA LCM, 2015). Os adolescentes que fazem uso de álcool são mais propensos a relatar relações casuais inesperadas (STARAS AS, et al., 2016).

Nos adolescentes do sexo masculino a prática de comportamento sexual de risco foi predominante diante do uso de substâncias psicoativas. Sendo que o preditor mais forte, estatisticamente, para o não uso de contracepção, foi o uso de drogas ilícitas (CASOLA AR, et al., 2017).

Não obstante, o início precoce da vida sexual é comumente associado ao uso de drogas (MIOZZO et al., 2013). A ocorrência de relações sexuais em detrimento destes fatores pode gerar uma experiência sexual 
traumática em um momento crítico do desenvolvimento, o que pode contribuir para concepção da normatização destes padrões de comportamento de risco (LIVINGSTON JA, et al., 2015).

Essas elevadas ocorrências de comportamento sexual de risco, revelada a partir do número de parceiros sexuais, nos adolescentes, esteve associada à idade, sexo, escolaridade materna, religião, idade da primeira ingestão de álcool e padrão de consumo de álcool nos últimos trinta dias e na vida.

Sendo que a "bebedeira" e a idade de iniciação alcoólica foram os principais fatores de risco para um comportamento sexual vulnerável (MOLA R, et al., 2017). Além disso, um nível socioeconômico mais baixo esteve diretamente associado ao baixo uso de preservativos, enquanto o consumo excessivo de álcool e 0 uso ilegal de drogas ilícitas foram associados de forma independente as relações sexuais inseguras (SANCHEZ ZM, et al., 2013).

\section{Infecções Sexualmente Transmissíveis em Adolescentes}

O comportamento sexual de risco pode resultar, além de uma gravidez indesejada, no contágio por alguma IST. Estudos alocados nesta categoria revelaram os fatores que estão comumente associados à incidência dessas infecções nesse grupo.

O desenvolvimento de atividades sexuais de maneira precoce e desprotegida entre os adolescentes, associada à multiplicidade de parceiros, acarreta um alto risco de IST, e de maximização dos índices de gravidez na adolescência.

São esses comportamentos sexuais de risco, principalmente a multiplicidade de parceiros, que criam uma rede que impulsiona a transmissão do Vírus da Imunodeficiência Humana (HIV) e da maioria das IST na população em geral (SHAYO FK e KALOMO MH, 2019). Uma das principais IST que mais geram preocupação é a AIDS, a qual apresentou maior tendência de crescimento entre homens adolescentes, heterossexuais, nos anos de 2004 a 2014, no Nordeste (CASTAÑO VA e ACEVEDO TLS, 2016).

Neste espectro, os fatores de risco mais frequentes, apontados para a ocorrência de IST em adolescentes, foram a sexarca precoce, ter relações frequentes e sem proteção, conhecimento ausente ou inadequado, violência física/emocional, multiplicidade de parceiros, parceiros mais velhos e relações com outros casais (WILSON CN e SATHIY AA, 2015; O'LEARY A, et al., 2015). Estiveram relacionados também fatores distintos, como residir em zona rural ou urbana, baixo nível de escolaridade, crenças religiosas, e condições socioeconômicas precárias (O'LEARY A, et al., 2015).

Preditores significativos foram apontados para a incidência das IST, principalmente naquelas adolescentes vítimas de violência por parceiro íntimo, ou com histórico de relação forçada (GONÇALVES H, et al., 2015; REIS AAC, et al., 2018). Essa alta prevalência de IST em adolescentes, que ainda estão iniciando a vida sexual, deve ser um alerta para a implementação de programas de alto impacto em saúde sexual (WILSON CN e SATHIY AA, 2015).

O comportamento sexual dos adolescentes mostrou-se estar estreitamente relacionado com 0 conhecimento que os mesmos detêm sobre o tema, o que influi diretamente nas suas condutas. Demais fatores que estão associados a tais práticas, em conjunto ou de forma isolada, produzem repercussões de risco para o comportamento sexual, expondo os adolescentes a diversas situações, que podem gerar consequências de grande impacto no contexto biopsicossocial.

\section{CONSIDERAÇÕES FINAIS}

Conclui-se que, a identificação dos fatores de risco citados neste estudo possibilita ações estratégicas, para intervenção nesse grupo possam ser conduzidas de forma mais efetiva, com vistas a obtenção de resultados significativos. Sugere-se que ações como a educação em saúde, o estabelecimento de vínculo efetivo do Programa Saúde na Escola com os profissionais da saúde da Unidade Básica de Saúde (UBS) e o estímulo para o envolvimento dos pais nas atividades, em conjunto com os adolescentes, possam ser realizadas a fim de alcançar à promoção da saúde dos adolescentes. 


\section{REFERÊNCIAS}

1. AGOSTINI A, et al. Comportamentos de risco entre adolescentes. Unoesc \& Ciência - ACHS, 2014; 5(1): 53-62.

2. BRASIL. Organização Pan-Americana da Saúde. Saúde e sexualidade de adolescentes: Construindo equidade no SUS. Brasília, DF: OPAS, MS; 2017.

3. CAMPOS MO, et al. Sexual behavior among Brazilian adolescents, National Adolescent School-based Health Survey (PeNSE 2012). Rev. bras. epidemiol. 2014; 17:116-130.

4. CASOLA AR, et al. Sex Differences in Contraception Non-Use Among Urban Adolescents: Risk Factors for Unintended Pregnancy. J Sch Health, 2017; 87(9):641-649.

5. CASTAÑO VA, ACEVEDO TLS. Prevalencia de infecciones de transmisión sexual y factores de riesgo para la salud sexual de adolescentes escolarizados, Medellín, Colombia, 2013. latreia. 2016; 29(1): 5-17.

6. CHINAZZO IR, et al. Comportamento sexual de risco em jovens: aspectos cognitivos e emocionais. Psico USF, 2014; 19(01): 1-12.

7. CLARK DA, et al. Sex, drugs, and early emerging risk: Examining the association between sexual debut and substance use across adolescence. PLoS One. 2020; 15(2): 1-18.

8. COELHO MEGM, et al. Fatores de risco para a saúde de adolescentes escolares: diferenças entre os sexos. Rev. enferm. UFPE. 2017; 11(supl.5). 2138-2144.

9. FARID ND, et al. Predictors of sexual risk behaviour among adolescents from welfare institutions in Malaysia: a cross sectional study. BMC Public Health. 2014; 14 (Suppl 03): 1-8.

10. GEBREYESUS $\mathrm{H}$, et al. Premarital sexual practice and associated factors among adolescents in the refugee camps in Tigray, northern Ethiopia. BMC Res Notes. 2019; 12(1): 415.

11. GONÇALVES H, et al. Sexual initiation among adolescents (10 to 14 years old) and health behaviors. Rev Bras Epidemiol., 2015; 18(01): 25-41.

12. GRAF DD, et al. Comportamento sexual de risco e fatores associados em universitários de uma cidade do Sul do Brasil. Rev. Saúde Pública. 2020; 41-54

13. GUTIERREZ EB, et al. Fatores associados ao uso de preservativoem jovens - inquérito de base populacional. Rev Bras Epidemiol. 2019; 22.

14. LINDBERG LD, et al. Prevalence of Sexual Initiation Before Age 13 Years Among Male Adolescents and Young Adults in the United States. JAMA Pediatr. 2019; 173(6):553-560.

15. LIVINGSTON JA, et al. Sexual risk at first coitus: Does alcohol make a difference?. J Adolesc., 2015; 43:148-58.

16. MAIA DAC, et al. Notificação de casos de HIV/AIDS em adolescentes portadores de HIV / Aids no Nordeste: série histórica entre os anos de 2004 a 2014. Revista Diálogos Acadêmicos. 2018; 7(1): 73-83.

17. MENDES KDS, et al. Uso de gerenciador de referências bibliográficas na seleção dos estudos primários em revisão integrativa. Texto contexto enferm. 2019; 28: 1-13.

18. MESQUITA JS, et al. Fatores de risco e de proteção entre adolescentes em relação às DST/HIV/AIDS. Rev. enferm. UFPE. 2017; 1(3): 1227-1233.

19. MIOZZO L, et al. Consumo de substâncias psicoativas em uma amostra de adolescentes e sua relação com o comportamento sexual. J Bras Psiquiatr. 2013; 62(2): 93-100.

20. MIRANDA PSF, et al. Sexual behaviors: study in the youth. Einstein. 2018; 16(3):1-7.

21. MOLA R, et al. Association between the number of sexual partners and alcohol consumption among schoolchildren. J Pediatr. 2017; 93(2):192-199.

22. MOURA LR, et al. A lacuna entre o conhecimento sobre HIV/AIDS e o comportamento sexual: uma investigação com adolescentes de Vespasiano, Minas Gerais, Brasil. Cad Saude Publica. 2013; 29(5): 1008-1018.

23. OCHEN AM, et al. Predictors of teenage pregnancy among girls aged 13-19 years in Uganda: a community based case-control study. BMC Pregnancy Childbirth. 2019; 19(1): 211.

24. O'LEARY A, et al. Associations between psychosocial factors and incidence of sexually transmitted disease among South African adolescents. Sex Transm Dis. 2015; 42(3):135-9.

25. OPAS. Organización Panamericana de la Salud. Acelerar el progreso hacia la reducción del embarazo en la adolescencia en América Latina y el Caribe. Informe de consulta técnica, Washington DC., EUA. 2018.

26. PATIAS ND, DIAS ACG. Sexarca, informação e uso de métodos contraceptivos: comparação entre adolescentes. Psico USF. 2014; 19(1): 13-22.

27. PIMENTEL JC, et al. Associação entre prática de atividade física e indicadores de comportamento sexual de risco em adolescentes. Rev Bras Ativ Fis e Saúde. 2013; 18(4): 493-500.

28. REIS AAC, et al. Desafios para as políticas públicas voltadas à adolescência e juventude a partir da Pesquisa Nacional de Saúde do Escolar (PeNSE). Ciênc. saúde coletiva, 2018; 23(9): 2879-2890.

29. REIS TG, OLIVEIRA LCM. Padrão de consumo de álcool e fatores associados entre adolescentes estudantes de escolas públicas em município do interior brasileiro. Rev Bras Epidemiol. 2015; 18(1): 13-24. 
30. SANCHEZ ZM, et al. Sexual behavior among high school students in Brazil: alcohol consumption and legal and illegal drug use associated with unprotected sex.Clinics. 2013; 68(4): 489-494.

31. SASAKI RSA, et al. Comportamento sexual de adolescentes escolares da cidade de Goiânia, Goiás. Rev Bras Epidemiol. 2014; 17: 172-182.

32. SHAYO FK, KALOMO MH. Prevalence and correlates of sexual intercourse among sexually active in-school adolescents: an analysis of five sub-Sahara African countries for the adolescent's sexual health policy implications. BMC Public Health. 2019; 19(1): 1285-1293.

33. SOUZA MT, et al. Revisão integrativa: o que é e como fazer. Einstein. 2010; 08(01): 102-6.

34. STARAS SA, et al. Increased Partner Risk Characteristic Among Adolescents Using Alcohol In the Moment. Sex Transm Dis. 2016; 43(9): 537-41.

35. TAQUETTE SR. Epidemia de HIV/Aids em adolescentes no Brasil e na França: semelhanças e diferenças. Saúde Soc. $2013 ; 22(2):$ 618-628.

36. VISALLI G, et al. Knowledge of sexually transmitted infections and risky behaviours: a survey among high school and university students. J Prev Med Hyg. 2019; 60(2): E84-E92.

37. WILSON CN, SATHIY AA. Associated Risk Factors of STIs and Multiple Sexual Relationships among Youths in Malawi. PLoS One. 2015; 10(8): 1-13. 